\title{
DESIGN ISSUES FOR THE ILC POSITRON SOURCE*
}

\author{
V. Bharadwaj, Y. Batygin, R. Pitthan, J. Sheppard, H. Vincke, J. W. Wang, SLAC, Stanford, CA \\ 94309, U.S.A., J. Gronberg, W. Stein, LLNL, Livermore, CA 94550, U.S.A
}

\section{Abstract}

A positron source for the International Linear Collider (ILC) can be designed using either a multi-GeV electron beam or a multi-MeV photon beam impinging on a metal target. The major design issues are: choice of drive beam and its generation, choice of target material, the target station, positron capture section, target vault and beam transport to the ILC positron damping ring complex. This paper lists the ILC positron source requirements and their implications for the design of the positron source. A conceptual design for the ILC is expected to be finished in the next two years. With emphasis on this timescale, source design issues and possible solutions are discussed.

\section{ILC PARAMETERS}

The ILC is a proposed $\mathrm{e}^{+} \mathrm{e}^{-}$linear collider with nominal center-of-mass energies $0.5-1.0 \mathrm{TeV}$ [1]. Design efforts for such colliders have been ongoing for some time using normal-conducting (NC) and super-conducting (SC) RF. Recently SC technology has been chosen as the preferred technology for ILC. Two conceptual designs [2],[3] exist for such a "cold" linear collider and form the basis for optimizing the design of the ILC. Table 1 shows the major parameters of the collider from the US Linear Collider Technical Options Study (USLCTOS) [2].

Table 1: USLCTOS SC Collider Parameters

\begin{tabular}{|l|c|c|}
\hline \multicolumn{1}{|c|}{ Parameter } & Ref. design & Upgrade \\
\hline Beam Energy $(\mathrm{GeV})$ & 250 & 500 \\
\hline RF gradient $(\mathrm{MV} / \mathrm{m})$ & 28 & 35 \\
\hline Two-Linac length $(\mathrm{km})$ & 27.00 & 42.54 \\
\hline Bunches $/$ pulse & 2820 & 2820 \\
\hline Particles $/$ bunch $\left(10^{10}\right)$ & 2 & 2 \\
\hline Beam pulse length $(\mu \mathrm{s})$ & 950 & 950 \\
\hline Pulse/s $(\mathrm{Hz})$ & 5 & 5 \\
\hline Luminosity $\left(10^{33} \mathrm{~cm}^{-2} \mathrm{~s}^{-1}\right)$ & 25.6 & 38.1 \\
\hline Average beam power $(\mathrm{MW})$ & 22.6 & 45.2 \\
\hline Total number of klystrons & 603 & 1211 \\
\hline Total number of cavities & 18096 & 29064 \\
\hline
\end{tabular}

\section{POSITRON SOURCES SCHEMES}

From Table 1 it is seen that the ILC positron source needs to deliver $1 \mathrm{~ms}$ long bunch trains containing 2820 bunches with $2 \times 10^{10} \mathrm{e}^{+} /$bunch at $5 \mathrm{~Hz}$ to the positron main linac. In comparison it is noted that the SLC positron source produced single bunches with $4 \times 10^{10} \mathrm{e}^{+} /$bunch at $120 \mathrm{~Hz}$, a factor of almost 60 less positron flux and 1500 less in pulse intensity. This increase in needed positrons requires new ideas and $R \& D$ to implement.

* supported by United States Department of Energy through contracts DE-AC02-76SF00515 at SLAC and W-7405-Eng-48 at LLNL .
To create positron bunches of the required quality for use in the ILC main linac, the following steps are needed. First a suitable drive beam - in type, energy, intensity, beam size and pulse structure - is generated. This beam impinges a target and generates electromagnetic showers. The positrons produced in the showers are then captured, separated from the other shower products and transported to one or more positron damping rings whose function it is to "cool" the bunches in phase space so that they are suitable for injection into the main linac.

There are three main schemes suggested for positron production for the ILC. The first - called "conventional" because all current positron sources are based on this method - uses a multi-GeV electron beam impinging on a thick, typically many radiation lengths, high- $Z$ target. The second and third schemes both use multi-MeV photons impinging on a thin, typically a fraction of a radiation length, target to pair-produce the positrons. The biggest advantage of using a photon drive beam is that if the photons are polarized, the resulting positrons can have significant polarization. Colliding the already polarized electrons with polarized positrons is believed to greatly enhance the physics potential of the ILC [4].

The second scheme - "undulator-based" - was developed in DESY for the proposed TESLA [3] linear collider and generates the multi-MeV photons by passing a very high (few hundred $\mathrm{GeV}$ ) energy electron beam through a long short-period undulator. To save on linac costs, the electron pulse trains used for collisions are also used for positron generation. As seen in Figure 1, the electron beam is extracted from the electron main linac, passes through a long undulator. The electron beam is then re-injected back into the main linac and the photons generated in the undulator are transported to the target system. It is apparent that the electron and positron systems are coupled and this will lead to increased ILC commissioning time and loss in integrated luminosity. Various schemes to mitigate these effects have been proposed and need to be fully evaluated.

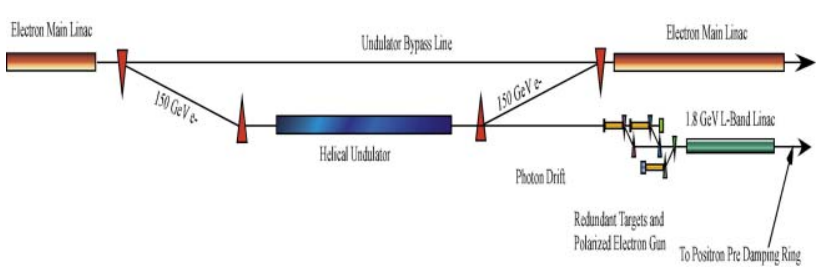

Figure 1: Undulator-based Positron Production Drive Beam Generation

The third scheme - "Laser-Compton scattering" generates the multi-MeV photons by Compton backscattering a very high power laser beam on a multi-GeV electron beam [5]. Although this scheme avoids the use of 
the ILC main linac, building the needed very high intensity laser and/or complex laser light distribution systems is problematic. Because the design is not as mature as the first two schemes the "Laser-Compton" method will not be discussed any further in this paper.

Drive beam parameters for the "conventional" and "undulator-based" positron sources are shown in Table 2. For the sake of discussion, the parameters are taken from the USLCTOS [2] - the TESLA design has qualitatively similar numbers. It is seen that for the "conventional" source case the electron beam energy is chosen to be 6.2 $\mathrm{GeV}$, a number that came from the Next Linear Collider (NLC) design. The positrons produced are linear with drive beam power, so that a lower energy, higher intensity drive beam can also work if this is advantageous for other reasons. The table emphasizes the undulator parameters needed to generate the multi-MeV photons. The photons generated in an undulator are not mono-energetic but have a known energy distribution and the photon energy quoted for such systems is the cut-off energy of the fundamental mode of the photon distribution.

Table 2: Drive Beam Parameters

\begin{tabular}{|c|c|c|}
\hline Parameter & $\begin{array}{c}\gamma \\
\text { beam }\end{array}$ & $\begin{array}{c}\mathrm{e} \\
\text { beam }\end{array}$ \\
\hline Electron Drive Beam Energy $(\mathrm{GeV})$ & 153 & 6.2 \\
\hline Beam Energy Loss $(\mathrm{GeV})$ & 4.9 & - \\
\hline Beam Energy Spread In \%() & 0.5 & - \\
\hline Beam Energy Spread Out (\%) & 0.46 & - \\
\hline Additional linac length $(\mathrm{m})$ & 170 & 230 \\
\hline Undulator length $(\mathrm{m})$ & 150 & - \\
\hline Undulator insertion length (m) & 790 & - \\
\hline Positron source length $(\mathrm{m})$ & 450 & 450 \\
\hline Photon energy $(\mathrm{MeV})$ & 10.7 & - \\
\hline Undulator type & $\begin{array}{c}\mathrm{K}=1 ; \\
\text { helical }\end{array}$ & - \\
\hline Undulator field $(\mathrm{T})$ & 1.07 & - \\
\hline Undulator period $(\mathrm{cm})$ & 1 & - \\
\hline Undulator full gap (mm) & 6 & - \\
\hline Positron yield ${ }^{\dagger}$ & 1.5 & 1.5 \\
\hline
\end{tabular}

The photon drive beam energy is a function of the electron drive beam energy and the undulator parameters, typically $10-30 \mathrm{MeV}$. This energy needs to be carefully optimized considering the constraints both of undulator technology and the electron beam parameters needed for ILC physics.

\section{POSITRON SOURCE OVERVIEW}

The positrons produced in any of the above schemes have to captured and transported to the ILC positron damping ring complex. All three schemes for positron production use essentially the same method for positron capture and transport. Figure 2 shows a schematic for a generic positron source capture layout.

The incoming drive beam ( $\mathrm{e}^{-}$or $\gamma$ ) hits a target, typically 4.5 radiation lengths of a tungsten-rhenium alloy for an electron beam and 0.4 radiation lengths of a titanium alloy for a photon drive beam. The produced positrons are focused and matched by a solenoidal field and captured and accelerated by an L-band RF system. At about $250 \mathrm{MeV}$, the positron beam is separated from the electrons and photons resulting from the electromagnetic shower and further accelerated and transported to the damping ring complex. The matching solenoidal field generated by a so-called "Adiabatic Matching Device" (AMD) - is typically 5.0 Tesla at the target decaying down to 0.5 Tesla in about $20-50 \mathrm{~cm}$ and then remaining at that value until the beam energy reaches the $250 \mathrm{MeV}$ beam separation point.

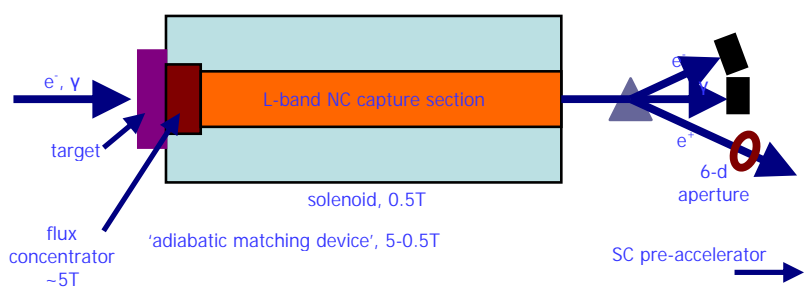

Figure 2: Generic Positron Source

The major technical issues in the design of the positron source target system are the target, the AMD, the L-band capture RF, the design of the target vault and source availability and maintenance.

\section{POSITRON TARGET}

Once the drive beam for the positron source has been generated it impinges on a metal target. For an electron drive beam, thick targets with a high- $Z$, high-density are needed. These materials have very short radiation lengths and hence the target is physically thinner for a given number of radiation lengths, reducing positron emittance growth due to multiple scattering. A tungsten-rhenium alloy (W76Re24) has been the target material of choice. In the photon drive beam case, a target only a fraction of a radiation length is needed and lower density materials can be considered. This allows for the choice of stronger materials such as titanium alloys. Table 3 shows the USLCTOS parameters relevant to the design of positron source target stations (note that in the $\mathrm{e}^{-}$beam case there are two target stations).

A reasonable target system criterion is to design for a lifetime of two years with the expectation that it will be replaced every year. The most important issues in the choice of target material and the design of the target are peak energy deposition, average energy deposition and chronic target degradation due to radiation. In designing the details of the positron target, the SLC experience provides an experimental working point for estimating allowed energy depositions. The SLC drive beam pulses consisted of single bunches of $4 \times 10^{10}$ electrons at $30 \mathrm{GeV}$ and $120 \mathrm{~Hz}$. The average SLC beam power was $24 \mathrm{~kW}$ with about $20 \%$ energy absorption in the target. The target moved approximately $0.4 \mathrm{~m} / \mathrm{s}$. For the ILC, the drive beam average power is of order a few hundred kilowatts factor of 10-15 more than SLC - with beam sizes of order 
$1 \mathrm{~mm}$. The pulse energy is almost three orders of magnitude greater. If the beam is parked on a single spot, the beam power is sufficient to destroy a metal target as a result of damage from target heating by the beam and the associated "shock" stress. These effects are mitigated by fast target motion. Typically the positron target is a rotating disk or "propeller" one meter or more in diameter moving at 100 's $\mathrm{m} / \mathrm{s}-$ i.e. tens of cm's in the $1 \mathrm{~ms}$ ILC pulse duration. If the target cannot be made to move at these speeds, multiple parallel target systems, as indicated in Table 3, can be used and in the case of the conventional source longer drive beam pulses can be considered.

Table 3: Positron Source Target Parameters

\begin{tabular}{|l|c|c|}
\hline Parameter & $\boldsymbol{\gamma}$ beam & e- beam \\
\hline Pulse energy on target [kJ] & 44 & 28 \\
\hline Average power per target [kW] & 222 & 140 \\
\hline Spot size on target [mm] & 0.75 & 2.5 \\
\hline Target material & $\begin{array}{c}\text { Ti- } \\
\text { alloy }\end{array}$ & $\begin{array}{c}\text { W76 } \\
\text { Re24 }\end{array}$ \\
\hline Target thickness [r.l.] & 0.4 & 4.0 \\
\hline Target energy absorption [\%] & 8 & 14 \\
\hline Target Radius [m] & 0.80 & 1.0 \\
\hline Revolution Rate [rpm] & 1200 & 1200 \\
\hline Pulsed Temperature Rise $\left({ }^{\circ} \mathrm{C}\right)$ & 410 & 256 \\
\hline Number of targets/spares & $1 / 1$ & $2 / 3$ \\
\hline
\end{tabular}

Neutron photo-production thresholds in target materials vary as $\mathrm{A}^{(-1 / 3)}$ and are in the range of $10 \mathrm{MeV}$. This favors using lower energy photons and lower- $Z$ target material to minimize target activation. With the appropriate choice of photon energy and target material the activation is expected to be much less than in the conventional case.

There exists a large body of knowledge on the effects of chronic radiation damage of materials. Nonetheless the allowed radiation damage for positron targets is not very well understood at present. Radiation damage effects are more severe for the titanium alloys used for the photon drive beam target and have to be mitigated in the design.

\section{ADIABATIC MATCHING DEVICE}

Immediately downstream of the target, a solenoidal magnetic field that starts at $5.0 \mathrm{~T}$ and goes down to $0.5 \mathrm{~T}$, matches the phase space of the positrons coming out of the target to the L-band capture RF system. Building a DC normal conducting solenoid with this peak field is not feasible. In the SLC this field was achieved using a pulsed "flux concentrator" (FC), a $20 \mathrm{~cm}$ long 12-turn solenoid with a conical beam pipe with a $1 \mathrm{~cm}$ aperture at its upstream end. This device was pulsed with a $16 \mathrm{kA} 100$ $\mathrm{kHz}$ half-sine wave and provided the required magnetic field profile for the SLC single bunch pulse train.

Extrapolating the "flux concentrator" concept for the much longer ILC pulse train will be challenging. An alternate approach is to use a large $-\sim$ one meter diameter - shielded superconducting DC solenoid. Calculations using FLUKA [6] show that the energy deposition due to the beam in such a solenoid can be reduced to less than 1 $\mathrm{W}$ out of the few hundred $\mathrm{kW}$ of incoming beam power. In addition the target material will see the full 5T field instead of the 1T that "leaks" out of the FC - and calculations show that this will improve positron yield significantly.

\section{L-BAND RF CAPTURE SECTION}

The positrons produced at the target are captured in an L-band RF section that is immediately downstream of the target and within the solenoidal magnetic field. Because of its location immediately after the positron target, the first part of this RF system cannot be superconducting. The main issues in the design of the L-band RF is how much gradient can be generated in normal conducting Lband RF systems and at what point do the beam losses become small enough to switch to a superconducting RF system. Positron yield calculations indicate that gradients greater than $10 \mathrm{MV} / \mathrm{m}$ - preferably $15 \mathrm{MV} / \mathrm{m}$ - are needed for efficient capture of the positrons. This along with the requirement of a few percent duty cycle and the heating [7] due to the drive beam make these cavities a challenge to design [8].

\section{TARGET VAULT}

At this early stage of positron source design for the ILC only minimal attention has been paid to the target vault design. Given that the positrons are made by impinging beam onto a target, the target environment will become very radioactive, especially in the conventional schemes. This along with the extreme temperatures and mechanical constraints of the spinning target with water and vacuum feedthroughs make for a very difficult design. Emphasis has to be given to both making the target very reliable and easy to maintain and to designing the vault to make it easy to repair and replace the target as necessary.

\section{REFERENCES}

[1] M. Tigner, "The International Linear Collider (ILC), Organization and Plans" proceeding, this conference.

[2] "Accelerator Technology Options Report", 2004, URL http://www.slac.stanford.edu/xorg/accelops/

[3] “TESLA Technical Design Report," 2001, URL http://tesla.desy.de/new_pages/TDR_CD/start.html

[4] "Power Collaboration Web Page", URL http://www.ippp.dur.ac.uk/Research/polarisation.html

[5] K. Dobashi et al., Design of Polarized-Positron Generation System, NIM A455, 32-35, 2000

[6] A. Fasso, A. Ferrari, J. Ranft, P. Sala, "FLUKA" URL http://www.fluka.org/

[7] V. Bharadwaj et al., "Heat Deposition in Positron Sources at the ILC", proceedings this conference.

[8] J. W. Wang et al., "Studies of Room Temperature Accelerator Structures for the ILC Positron Source", proceedings this conference. 